\title{
Incorporating the Whoqol-Bref Questionnaire in Assessing the Quality Of Life amongst Students in a Private Tertiary Institution in Nigeria
}

\author{
${ }^{1 *}$ Igwilo.A.I, ${ }^{2}$ Igwilo.U.U, ${ }^{3}$ Adigun.A, ${ }^{4}$ Padonu.M.K.O, ${ }^{5}$ Oridota.F.,${ }^{3}$ Hassan.F. \\ ${ }^{I}$ Oncology Unit, Department of Radiology, Lagos State University Teaching Hospital; \\ ${ }^{2}$ Lagos Mainland Local Government Primary Health Care Centre, Lagos; \\ ${ }^{3}$ Lagos University Teaching Hospital, Idiaraba, Lagos; \\ ${ }^{4}$ College of Health Sciences, Igbinedion University, Okada,Edo State \\ ${ }^{5}$ Department of Community Medicine, College of Medicine,University of Lagos,Idiaraba,Lagos
}

\begin{abstract}
It is the norm/belief that being in the upper socio-economic class parallels a higher quality of life. In 2008, during the Medical outreach of the annual Health week held by the Igbinedion University medical students' Association, this hypothesis was tested.

To obtain a basic assessment of QOL among the students in this rural location, determining the factors which affect the Quality of life and providing, if any, strategies to improving QOL among students in the school as well as such schools in rural locations globally.

This study is a cross-sectional descriptive one in which a total of 82 students were selected by simple random sampling technique were subjected to self-administered closed-ended 24-numbered semi-structured questionnaire on the basis of the standardized SF-12 along with other factors under consideration, incorporated Of the 82 students randomly selected, there was also a statistical significant association between the factors understudy and quality of life.

There is a need for a public awareness of the ups and down determinants of health especially where the economy, career and health divide meets and strategies thought and reproduced to improve the QOL among students of institutions sited especially in rural communities in Nigeria and the world in general.
\end{abstract}

Keywords :Quality of Life(QOL), Igbinedion University, SF-12, students, Association

\section{Introduction and Rationale}

Students are engaged in many demanding tasks, thus the need for proper well-being of both mind and body cannot be overemphasized.

It is commonly believed that certain factors determine one's quality of life amongst students such as diet patterns, monthly income, gender and surprisingly even course and source of meals, considering the school being situated in a rural community (Okada) in Edo state, Nigeria, which is about 3000 miles from the nearest city-Benin. Okada is situated in Ovia North-East area of Edo state in Southern Nigeria.

It is noteworthy that a study done to examine and compare health status between rural \& urban adults in Kentucky, U.S.A showed that although the health status of rural \& urban adults is generally similar, the rural elderly have significantly worse health status than their urban counterparts(1).

In addition, another study which explored the effects of mental status and gender on the subjective quality of life in Korea using five separate scales to measure the quality of life. These scales included a singleitem happiness scale, two negative affect scale, a modified version of Life in General Scale developed by Campbell et al(1976), and a scale measuring the sense of personal competence. The result indicates that gender is a stable predictor of the quality of life regardless of one's marital status; men are better off than women(2).

A study carried out to determine and compare the nutritional status of children attending urban \& rural public primary schools in Ife Central Local Government Area of Nigeria, showed that the nutritional indices (weight for height, weight for age and height for age) were found to be significantly lower among the rural pupils than urban pupils $(\mathrm{P}<0.001$ in each case $)(3)$. Thus there is a positive correlation between nutritional (health) status and community.

Igbinedion University is a private tertiary institution founded in 1999 by the Esama of Benin, Chief Sir. Gabriel Igbinedion, who is also the chancellor of the Institution. The School currently has a population of over 2000 students and offers courses in both undergraduate and postgraduate schools. The annual tuition is above N300, 000 (Three hundred thousand Naira). Undergraduate faculties currently include Health sciences, Engineering, Law, Natural \&Applied sciences and Arts \& Social Sciences. Apart from the school-owned café,other notable restaurants in the school include Mama Cass/Café (a bit expensive),T\&B and Chills. Therefore, most of the students are children/wards of parents who belong to the upper socioeconomic class as 
these can afford such tuition costs. Invariably, this presents Igbinedion University students in a different plane from their counterparts in other universities/institutions (both private \& Federal) especially in terms of specific health status factors such as monthly income \& diet patterns.

Conclusively, the framework of this study is to evaluate the relationship between the quality of life AND sex, course being studied, monthly income, meal pattern \& source of meals.

\section{Methodology}

This is a cross sectional descriptive study carried out during the annual Igbinedion University Medical Association (IUMSA) health week in June, 2008, which was flagged off by a medical outreach in which semiconstructed questionnaires were randomly (simple random sampling)distributed among the students and the results collated. A written consent from the School Authorities and an oral consent were received from each respondent before the study commenced.

\subsection{Objectives}

The study was done to:

$>$ To obtain a knowledge of the relative quality of life (QOL) among the students.

$>$ To group the students based on a stratified random sampling technique based on certain parameters to determine the QOL in the individual strata.

$>$ To determine if there is an existing relationship between QOL and the individual stated parameters.

$>$ To provide solutions to the falling QOL indices while making suggestions to improving QOL among Private tertiary institution-attending students in such rurally-located institutions globally.

The semi structured 24-numbered questionnaire was culled from the SF-12 health survey (Physical Component \& Mental Component Scores) of which 12 of the questions were from SF-12, while the remaining 12 were used to get the relationship between the SF-12 response and the social determinants of health variables (sex, course, diet pattern, source of meals and monthly income).

A data obtained from a cross sectional health status survey among 82 students of the school in June 2008, obtained by a simple random sampling method, using a closed-ended 24-numbered questionnaire, which included the SF-12, was self-administered to each student to ascertain information such as age group, sex, faculty, Diet patterns, source of meals \& average monthly income. The SF-12 of each person was also calculated to obtain the quality of life based on its two components (PCS \& MCS), comprising the eight subscales-Physical functioning, role functioning (physical \& emotional), bodily pain, general health, vitality, social functioning and mental health. Using standard methods, the two component summary scores were calculated based on the response of each subscale-represented question. Each subscale is standardised using a Zscore transformation and assembled to estimate the individual physical and mental summary scores.

Although, all eight subscales contributes to both summary scores, the physical component summary score is predominantly made up of the physical functioning subscale; the physical role function, vitality and general health subscale contribute minimally. Whereas the mental component summary score is made up predominantly by the mental health subscale followed by emotional role functioning, social functioning and the vitality subscale also. The summary scores range from 0-100 and higher scores indicate a better quality of life.

\subsection{Statistical analysis}

For each student, the PCS and MCS scores were obtained based on their responses to the relevant questions. The frequency, the mean, variance and the standard deviation were obtained as well as the and monthly income of each student, using the Chi squared test $\left(\mathrm{X}^{2}\right)$. To analyse the collated data, the answers to the questions for each questionnaire were fed into a program web link -http://www.SF-36/demos/SF-12.html, which converted them into scores (numbers), and an average was obtained in each category, which was used to classify each student involved in the study, into positive (above average) or negative (below average) in each subcomponent of each category/variable.

\section{Results/Findings}

A total of 82 questionnaires were analysed in this study.

Following the analysis of data above, the mean Physical component score (PCS) was 48.1, and the mean mental component score (MCS) was 47.8.Of the physical component, the variance was 70.7 and standard deviation 8.41,while that for mental component was 83.2 and 9.1 respectively.

The relationship between the QOL components (PCS \& MCS) was found to vary significantly with the sex, course, diet pattern, source of meal and monthly income at $\mathrm{p}=0.05$. 


\subsection{Basic Demographics}

\subsubsection{Gender}

Females were found to have a relatively higher quality of life (57.8\%) than males $(42.2 \%)$ in the mental subcomponent; however, both males and females had equal percentage in the physical sub component of the QOL.

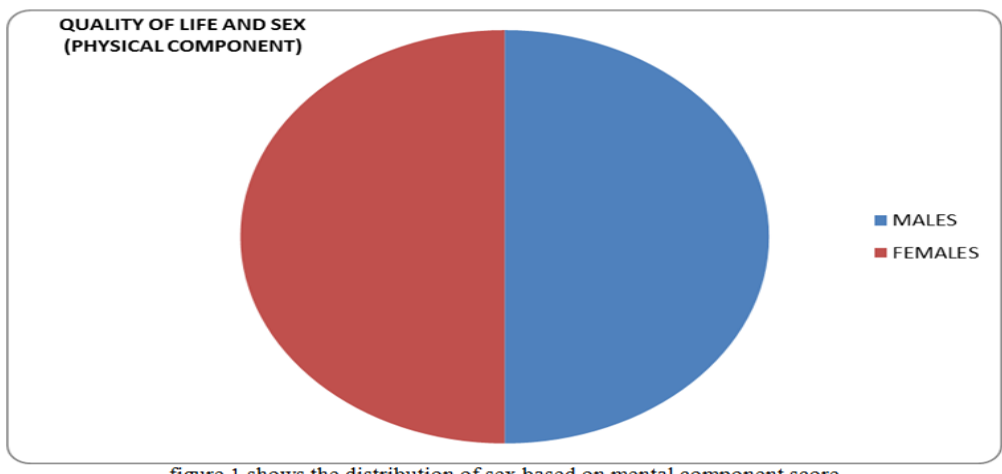

1 shows the distribution of sex based on mental component score

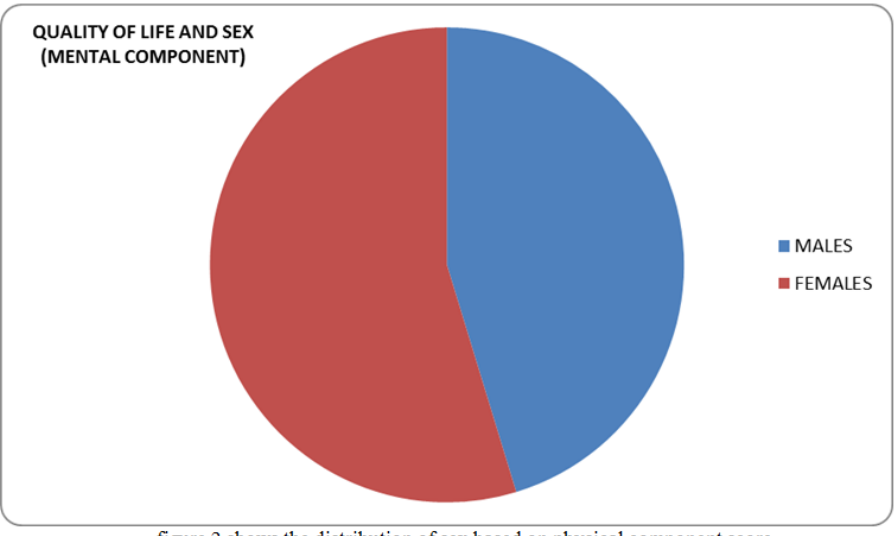

figure 2 shows the distribution of sex based on physical component score

\subsubsection{Course}

Respondents in health sciences, Engineering \& 'others' had the highest quality of life in the mental subcomponent,i.e.23.8\%,23.8\% \& 23.8\% respectively, while only Health sciences $(37.5 \%)$ had the highest in the physical subcomponent. Among the lowest were natural \& applied sciences $(11.9 \%)$ in the mental subcomponent and Law (5\%) in the physical component as shown below.

TABLE 1 shows the Distribution of Respondents into their respective courses based on their Physical component scores

\begin{tabular}{|l|l|l|l|l|l|l|l|}
\hline & $\begin{array}{l}\text { Health } \\
\text { Sciences }\end{array}$ & Law & Engineering & $\begin{array}{l}\text { Natural } \\
\text { \& } \\
\text { Applied } \\
\text { Sciences }\end{array}$ & Arts & Others & TOTAL \\
\hline POSITIVE & 15 & 2 & 7 & 6 & 5 & 5 & 40 \\
\hline NEGATIVE & 7 & 6 & 8 & 2 & 12 & 7 & 42 \\
\hline TOTAL & 22 & 8 & 15 & 8 & 17 & 12 & 82 \\
\hline
\end{tabular}




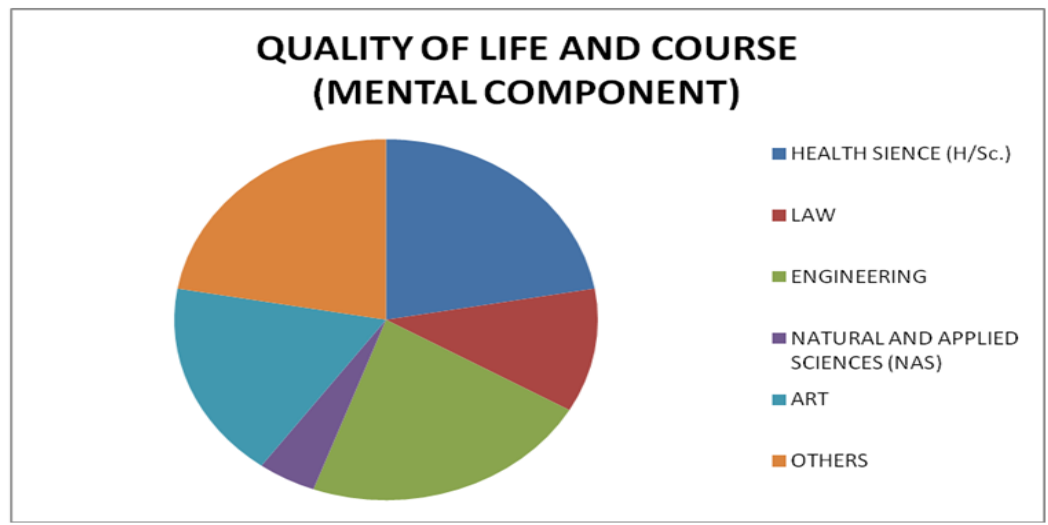

figure 3 shows the distribution of respondents' courses based on their mental component score

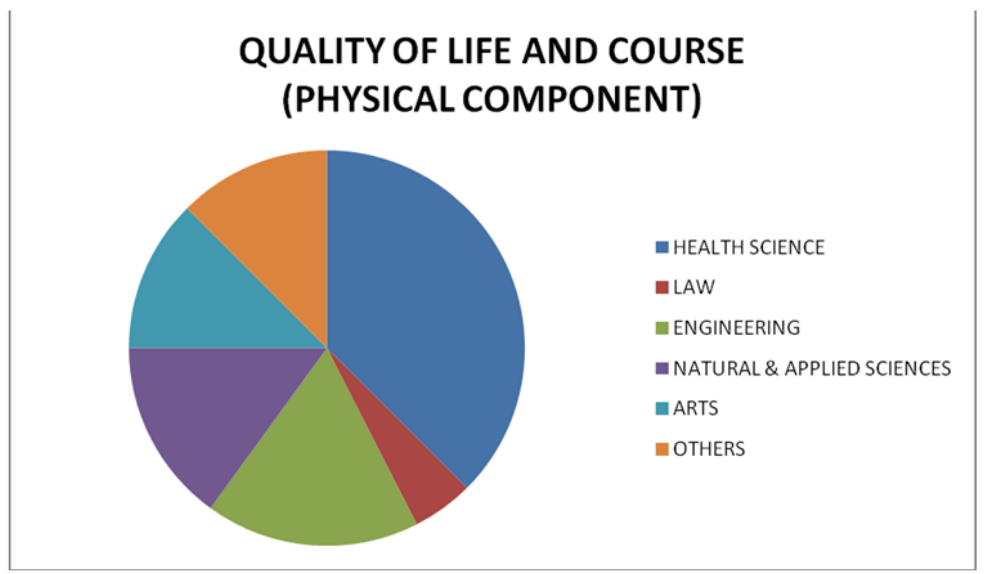

figure 4 shows the distribution of respondents courses based on their physical component score

\subsubsection{Frequent Meal Source}

Respondents who sourced their meals from the school-owned café (9.7\%) and Mama-café/cass (9.7\%) had the lowest mental component score while those who sourced their meals from multiple eatery joints $(21.9 \%)$ and 'self-cooking' (21.9\%) had the highest mental component score. It was also found that those who cooked their meals recorded the highest physical component score $(23.7 \%)$ while those who sourced their meals from 'chills' recorded the lowest physical component score.

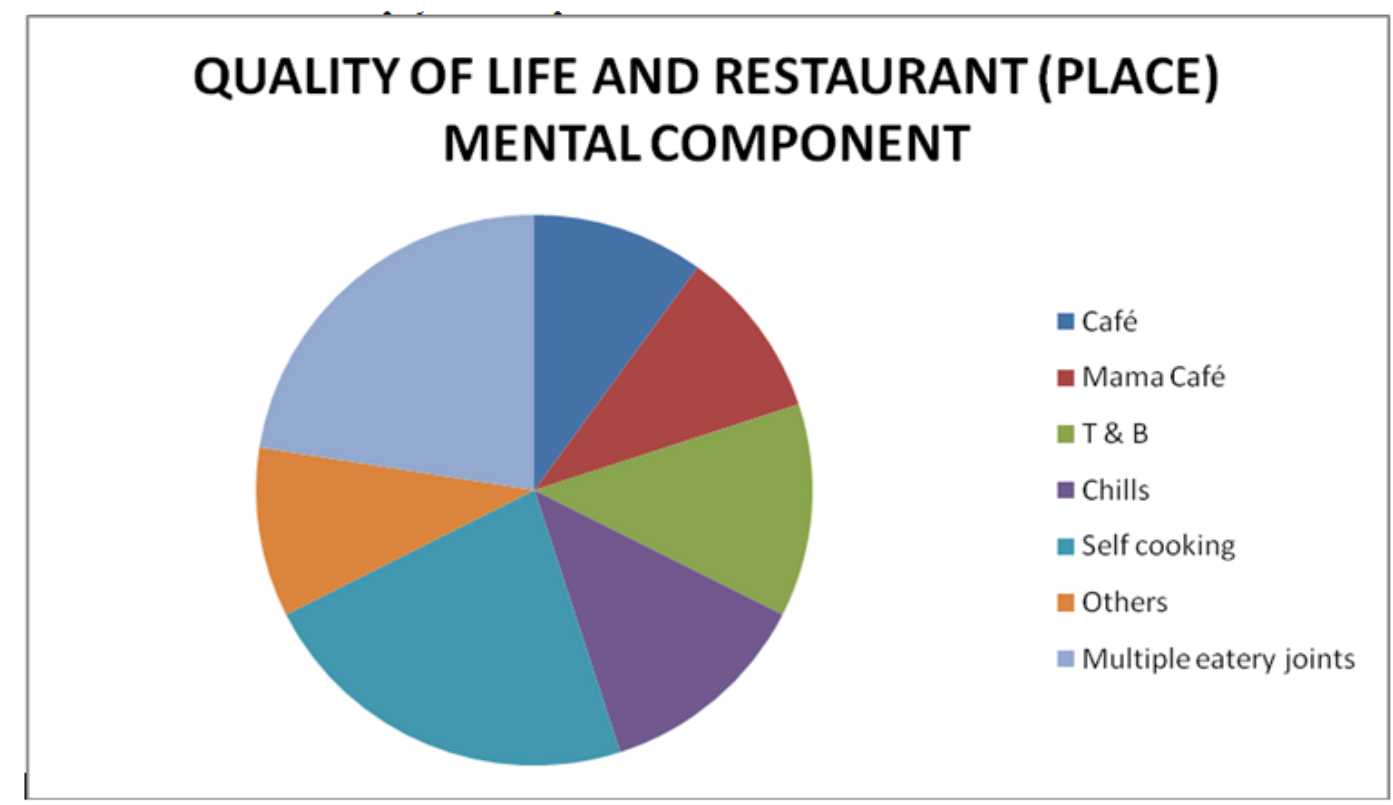

figure 5 shows the distribution of respondents meal source based on their mental component score 


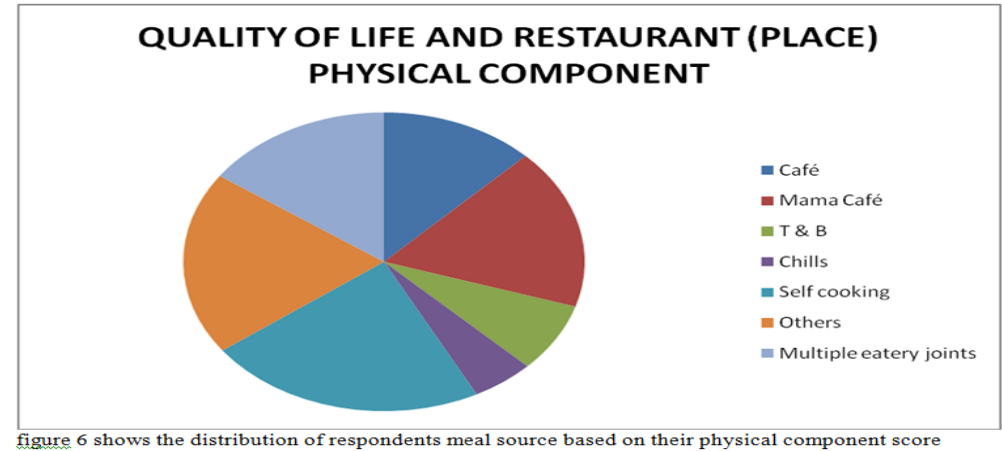

\subsubsection{Monthly Income}

Respondents who are on an monthly income of N10,000-N20,000 had the highest mental component score of $37.5 \%$ and those on an income $<\mathrm{N} 10,000$ recorded the lowest physical and mental component scores$2.8 \%$ \& 5\% respectively; While those on an income between N20,000- N30,000 had the highest physical component score of $38.9 \%$ as shown below:

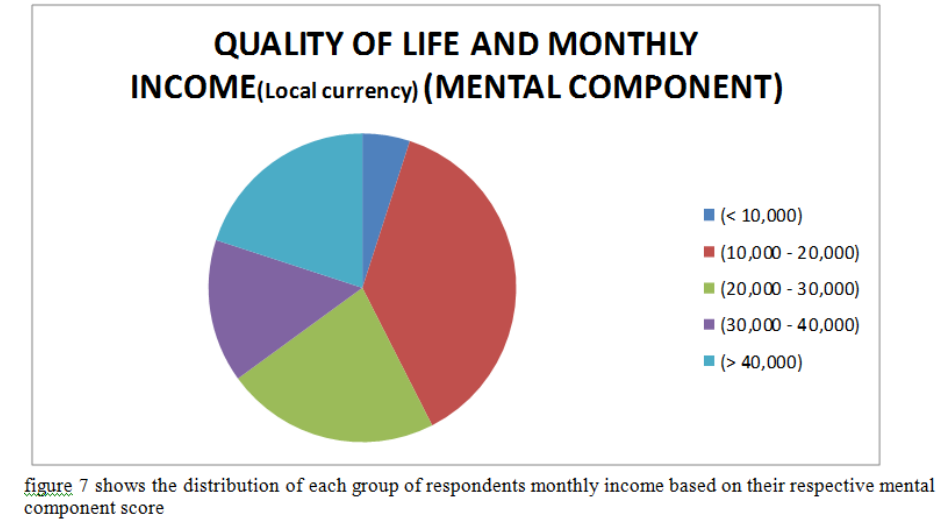
figure 7 shows the distribution of each group of respondents monthly income based on their respective mental
component score

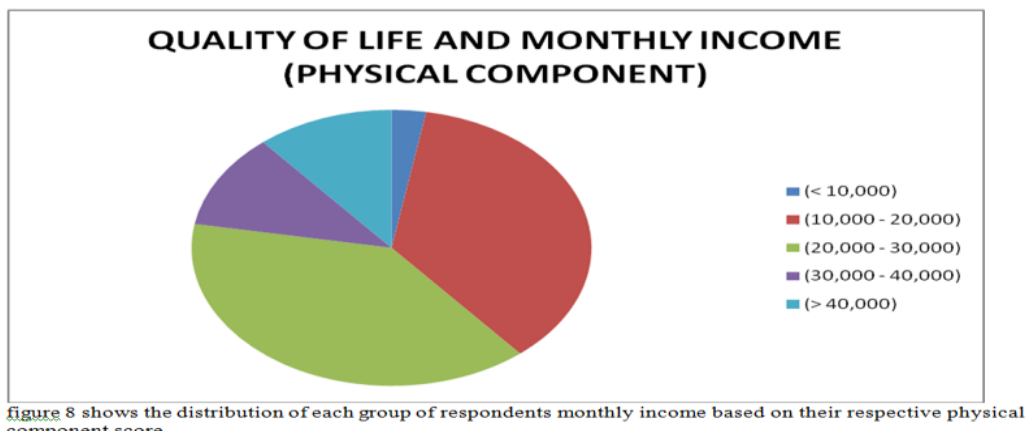

\subsubsection{Number of Meals}

Respondents who ate twice daily was found to have the highest mental and physical component score of $63.4 \% \& 53.8 \%$ respectively while those who ate four times daily had the lowest mental and physical component score of $2.4 \% \& 5.1 \%$ respectively, as shown below:

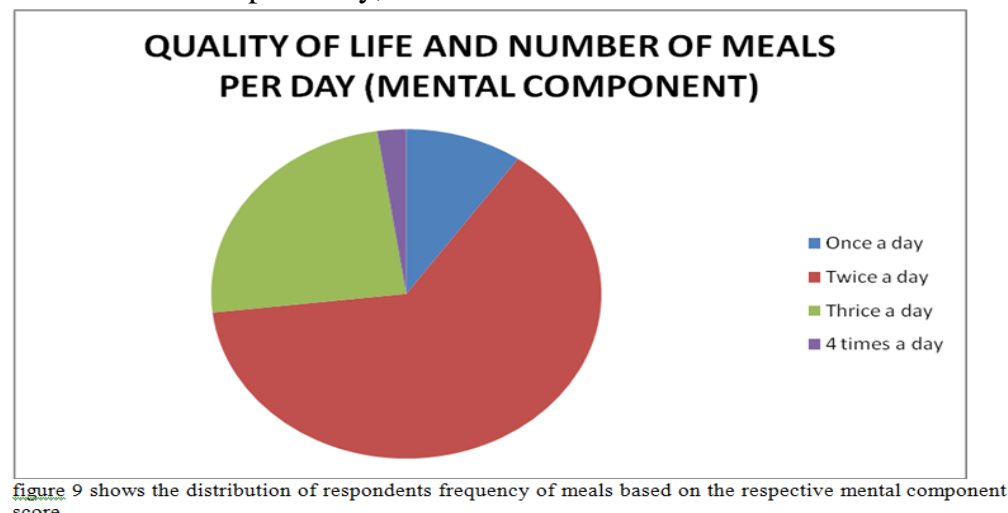




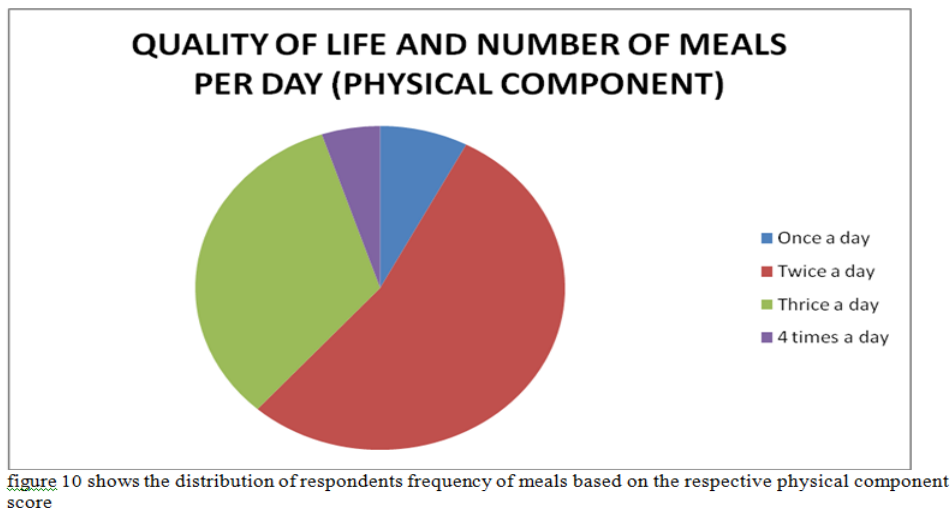

In summary:

* Between the quality of life (QOL) and sex, females had a relatively higher MCS score than their male counterparts. However, both sexes had equal PCS scores. For MCS and PCS, calculated $\mathrm{X}^{2}=3.2321$ and 0.0508 respectively at significance level of 0.05 ( $\mathrm{p}<\mathrm{a})$ at $\mathrm{df}=1$ (df-degree of freedom).

* In relation to course, students of the Health science faculty had the highest PCS and MCS scores with students of Law and Natural \& applied sciences tagging the lowest in PCS and MCS scores respectively. i.e. for MCS and PCS, calculated $\mathrm{X}^{2}=9.396$ and 10.1473 respectively at significance level of $0.05(\mathrm{p}<\mathrm{a})$ and $\mathrm{df}=5$.

* In relation to source of meals, students who cooked their meals surprisingly had the highest MCS \& PCS scores, compared to those who sourced their meals from school cafeteria \& Mama Cass AND chills who had the lowest MCS \& PCS scores respectively, contrary to the believed hypothesis amongst the students of the school that sourcing meals from an exquisite restaurant like Mama Cass should gain ground in one's health assessment. For MCS and PCS, calculated $X^{2}=7.2537$ and 8.6617 at significance level of $0.05(p<a)$ and $\mathrm{df}=6$

* Also students receiving a monthly income/allowance between N10,000- N20,000 and N20,000-N30,000 had the highest MCS and PCS while the < N10,000 monthly allowance group earned the lowest in both PCS \& MCS categories. For MCS and PCS, calculated $X^{2}=7.9047$ and 4.1606 respectively at significance level of $0.05(\mathrm{p}<\mathrm{a})$ and $\mathrm{df}=4$.

* Lastly, it was astonishing to find out that those who eat twice daily had the relatively highest PCS \& MCS scores compared to those who ate for times daily who at the bottom of the ladder in respect to QOL scores. For MCS and PCS, calculated $\mathrm{X}^{2}=1.235$ and 2.1425 respectively at significance level of $0.05(\mathrm{p}<\mathrm{a})$ and $\mathrm{df}$ $=3$.

These all indicates that there is an association between the social determinants and the QOL components beyond that attributable to chance.

\section{Discussion}

The World Health Organisation Quality of Life (WHOQOL) project was initiated in 1991. The aim was to develop an internationally cross-culturally comparable quality of life assessment instrument. It assesses the individual's perceptions in the context of their culture and value system, and their personal goals, standards and concerns. The WHO-BREF instrument comprises of 26 items, which measure the following broad domains: physical health, psychological health, social relationships, and environment. The WHOQOL-BREF is a shorter version of the original instrument that may be more convenient for use in larger research studies or clinical trials.(4)

The WHO's initiative to develop a quality of life assessment arose for a number of reasons. Firstly, in order to incorporate a quality of life and disease impact measurements into the measurement of health. Secondly, to develop a health status measurement this can be translated cross-culturally and globally. Thirdly, to minimize the increasingly mechanistic model of medicine this is currently concerned only with the eradication of disease and symptoms, by incorporating quality of life assessments in healthcare. Therefore, attention is also paid to this aspect of patients' wellbeing.(4) In other words, the need to develop a quality of life assessment arose from a need for genuinely international measure of quality of life and a commitment to the continued promotion of a holistic approach to health and healthcare.(5)

Health status is a broader view of which Quality of life is a factor used in assessing an individual's health status. In assessing the quality of life of any person, the two subcomponents of QOL is assessed i.e. the mental \& physical component scores obtained by the standardized SF-12 questionnaire preanswered program downloaded from the worldwide web. The need to obtain a value of health status amongst a group of people such as students of a randomly selected school and also assessing variable factors while obtaining the 
probability of a relationship between the independent (health status scores) and the dependent variables (factors),cannot be overemphasized as it will help in both enlightenment, Education, provision of better strategies to reach an acceptable/above average health status and probably in probing further (research)in analysing qualitatively the relationship between the variables.

The term quality of life is used to evaluate the general well-being of individuals and societies (6). Researchers at the University of Toronto's quality of life Research Unit define it as "The degree to which a person enjoys the important possibilities of his or her life".(7)

Health status may be defined as the level of health of the individual, group or population as subjectively assessed by the individual or by more objective measures. According to the World Health Organisation, quality of life is defined as an individual's perceptions of their position in life in the context of the culture and value systems in which they live and in relation to their goals, expectations, standards and concerns.(5) Within the field of healthcare, quality of life is often regarded in terms of how it is negatively affected, on an individual level, a debilitating illness that is not life-threatening, life-threatening illness that is not terminal, terminal illness, the predictable, natural decline in the health of an elder, an unforeseen mental/physical decline of a loved one, chronic, end-stage disease processes.(7)

Quality of life is a descriptive term that refers to people's emotional, social and physical well-being, and their ability to function in the ordinary tasks of living. Health-related QOL measures the impact of treatments and disease processes on these holistic aspects of a person's life. It is measured using designed \& tested instruments which measure people's ability to function in the ordinary tasks of living. QOL analysis aids investigating the social, emotional \& physical effects of treatment or disease from the patient's perspective; and determining the need for social, emotional \& physical support during illness. Unlike utility measures like the one used to calculate quality-adjusted life years (QALY), quality of life measures attempt to evaluate directly the impact of a disease or treatment on people's ability to function in life, not the value that they place on a particular health state.

Also, quality of life is the degree of well-being felt by an individual or group of people (subjective).It is intangible and consists of two components as stated earlier. The physical includes such things as health, diet and protection against pain and disease while the mental involves stress, worry, pleasure and other positive or negative emotional states.

Quality of life, a concept that depicts subjective well-being forms a contrast with objective indicators of life conditions. It is a topic that attracts the attention of many researchers. One of the reasons for this attention is that subjective wellbeing often does not correspond to the socioeconomic conditions of life. Studies generally indicate that family life and interpersonal relationships are more closely related with the internal, affective aspects of people's lives than are the external socioeconomic conditions, and therefore are better predictors of the quality of life.(2)

In a study previously mentioned, carried out to determine and compare the nutritional status of children attending urban and rural public primary schools in Ife Central Local Government Area (ICLGA) of Nigeria, it indicated that malnutrition (underweight, wasting and stunting) constituted major health problems among school children in Nigeria, particularly in the rural areas.(3)

Also,a study done to examine and compare health status between rural and urban adults are generally similar, the rural elderly have significantly worse health status than their urban counterparts.(1)

Quality of life is an important concept in the field of international development, since it allows development to be analysed on a measure broader than standard of living. Organisations, such as the World Bank, for example, declare a goal of "working for a world free of poverty" (8);

With poverty defined as a lack of basic human needs, such as food, water, shelter, freedom, access to education, healthcare, or employment. (9) In other words, poverty is defined as a low quality of life.

While quality of life should not be confused with the concept of standard of living which is based primarily on income, standard indicators of the quality of life will include not only wealth and employment, but also environment, physical and mental health, education, recreation, leisure time and social belonging surrounding an individual.(6)

Although it is impossible to predict with absolute accuracy the quality of life of a particular individual since the combination of attributes that lead one individual to be content is rarely the same for another individual, it can be assessed with some confidence that the higher average level of diet, shelter, safety, as well as freedoms and rights a general population has, the better overhaul quality of life it experiences. In the comparative study of Nutritional status of Urban and Rural Nigerian school children, it was stated that nutritional assessment in the community is essential for accurate planning and implementation of intervention programmes to reduce morbidity and mortality associated with under-nutrition.(3) Thus, the understanding of QOL is important in healthcare especially as decisions in relation to treatments \& investments in particular research projects, are closely related to a patient's quality of life i.e. it helps in making decisions between different treatments/habits, keep people informed on the effects (long-term/short-term) of these 
habits/treatments, monitor the success of the outcome from the individual's perspective and also plan \& coordinate healthcare educational campaign packages.

After analysing the completed semi-structured questionnaire (based on SF-12 health survey) obtained from the respondents randomly selected in the school, it was discovered that there was a probable relationship between the physical \& mental (psychological) component scores (health status) and the other social determinants such as sex, course, diet pattern, source of meals and income beyond that attributable to chance. In summary (as stated from the results above), it can be culled that females in the faculty/school of health sciences who ate their self-cooked meals two times daily and lived on a monthly allowance/income of between N10,OOO-N30,000 (£42.5-£127.6; \$85.5-\$256.4 using $£ 1=\mathrm{N} 235 \& \$ 1=\mathrm{N} 117$-exchange rate at the time of this study), had the highest QOL compared to male students of Law and Natural \& Applied Science faculties who ate out four times daily and lived on a monthly income/allowance of less than N10,000.

This disparity can be explained to a reasonable extent. Females generally are more domesticated generally added to the fact that those in health science faculty are also imbibed or possess a knowledge of what a balanced diet is, the essence and importance of such diets. Thus, they can differentiate a variety of foods based on their nutritional quantities and consume the appropriate variety for their body's nutritional requirement compared to males who are not that domesticated to invest their time in obtaining such knowledge, how much more preparing such foods themselves. Also, the cooking methods in various cafeteria \& restaurants vary and can affect the nutritive quality of the foods compared to foods that are self-cooked. For example, it is more preferable, health wise that one will go for fresh foods/ingredients, fruits \& vegetables in order to prepare his/her meal compared to restaurants that may purchase the processed/ready-made ingredients and use them to prepare their meals. They may also further process these foods further reducing the nutritional content of these foods. With a poor background knowledge of balanced diet and nutrient education combined with the fact that being in Law and Natural \& applied sciences faculties further draws the students from any form of basic knowledge of food \& nutrition, makes the acquisition and appropriate implementation of food \& nutrition skills difficult thus affecting the individual's general well-being \& health status especially as the quote goes "You are what you eat". Thus, poor nutritive intake will unequivocally lead to poor health status.

Furthermore, it was found that those eating twice daily having a higher health status than those eating four times daily. This is an astonishing finding. As students are involved in demanding tasks, it has therefore been found that consuming meals twice daily improves the well-being of the individual compared to four times daily. This may be due to the relative rate of food digestion, the quality of the food, amount of work, etc. Further qualitative research on this relationship can be analysed and the higher contributing factor found. In relation to this, student's monthly income/allowance of between N10, 000-N30, 000 is most likely to provide the adequate necessities in terms of feeding, personal effects and even adds a psychological well-being to an individual than those below N10, 000. This has a strong connection with to economy of the country \& area of the school's location. Okada, the location of the school is a rural community located over 3000 miles from the nearest city and because the traders who are also part of the rural community have to travel that far to buy food items (crops grown are not enough to meet the population needs) which are in turn sold to students on specific market days pre-specified by the community, the prices are usually inflated and sometimes excessively by few traders who feel that the students as they come from the upper socio-economic class have enough money to throw around and thus should be made to pay more than usual. All these contribute to a relative different price range of items sold here compared to the entire state and the country in general. And from the health status assessment, it can be recommended that a monthly allowance of between N10, 000-N30, 000 will be adequate for any student of a school in such a community, although other factors will have to come into play.

Although there are not so many studies comparing the parameters involved in this study, and amongst students, howbeit one study carried out in Iran amongst health and unhealthy students to obtain subjective wellbeing, depression, anxiety and stress ratings, the researchers found that the mean score for all satisfaction ratings (including quality of life rating) were found higher in healthy than unhealthy students, while the mean score for the three emotional states of stress, anxiety and depression were found higher in unhealthy than healthy students. Also, a significant correlation was found among all satisfaction and negative emotion ratings. In addition, the authors quoted Campbell, Converse \& Rogers (1976) as defining quality of life in terms of life satisfaction or satisfaction of needs, and Becker, Diamond \& Sainfort (1993) as defining it as "a person's sense of well-being that stems from satisfaction or dissatisfaction with the areas that are important to him/her.(10) Studies invested in objective measurements of quality of life such as this, is scanty. However, this study has been insightful and stimulating. We, the authors, hope that more research would be carried out in measuring such and more parameters in relation to quality of life, in various circles in order to appropriate amenities, aid decision-making processes, identify \& evaluate risks factors and inculcate healthy lifestyle habits were necessary. Interestingly, such studies are useful in almost every sector from politics, education, health, business and even at the grass root level e.g. family. More extensive quality of life studies done on a larger scale would prove useful. 


\section{Recommendation/Strategies}

The following can be of help in improving people especially student's quality of life (modifiable \& nonmodifiable factors):

- A basic balanced diet knowledge acquisition which could be brought about by posters and educational campaigns carried out in all the faculties of the school.

- Students should be advised to plan their time (time management) so as to incorporate cooking their meals, for example for a week because this will reduce both cost, make them aware of the need for fresh foods, aid student's time management and avoid the health-effects of over-processed foods/meals.

- Students who earn or receive less than the N10, 000 as their monthly income can help themselves by channelling a higher percentage of the money to highly nutritious foods they can afford rather than a low nutritive (balanced) meal, as well as also appropriating funds to other basic requirements. They may also incorporate an income-generating venture during their spare time to improve on their allowance monetary value.

\section{Conclusion}

From the above observations, it is paramount that efforts are placed on making students as well as the public aware of these findings, stimulating the interest of students globally in their health while making them aware of the risks.

This has exposed and stimulated keen interest, among students generally, in their health; There is a need for a public awareness of the ups and down determinants of health especially where the economy, career and health divide meets and strategies thought and reproduced to improve the QOL among students of institutions sited especially in rural communities in Nigeria and the world in general.

All these brings to mind a quote from Izaac Walton from some hundreds of years ago who said " Look to your health; and if you have it, praise God and value it next to conscience; for health is the second blessing that we mortals are capable of, a blessing money can't buy".

These are all for one goal; to attain an above-average assessment of health status of each individual in the world because at the end of the day, the quote will always be true, "Health is wealth".

\section{Acknowledgement}

We wish to acknowledge Prof. Ekanem of the Department of Community Medicine, College of Medicine, University of Lagos, who proof-read the entire rough draft of the project and recommended the work for international publication even at that stage post-corrections. This gave the team the moral boost which was much needed at the time.

\section{References}

[1]. Mainous.A.G. 3rd, Kohrs.F.P ; A comparison of Health status between rural and urban adults. J comm. Health;1995;20(5); 423-431

[2]. Suni Lee;Marital Status,Gender and Subjective Quality of life in Korea;Development and Society;1998;27(2);35

[3]. Oninla S, Owa J, Onayade A, Taiwo O: Comparative study of Nutritional status of urban and rural Nigerian School children. J Trop pediatr;2007;53(1);39-43

[4]. World Health Organisation ;WHOQOL study protocol; 1993;WHO (MNH 7PSF/93.9)

[5]. World Health Organisation;WHOQOL-BREF; Introduction, Administration, Scoring and Generic version of the assessment (Field Trial version); $1996 ; 1-18$

[6]. Gregory.D.,Johnston.R.,Pratt.G. et al;eds;"Quality of Life"; Dictionary of Human Geography (5 ${ }^{\text {th }}$ ed); Oxford: WileyBlackwell;2009; ISBN 978-1-4051-3287-9.

[7]. University of Toronto Quality of Life Research Unit:”Quality of Life: How Good is Life for You?”;Retrieved October 14,2009.

[8]. The World Bank;'The World Bank";2009; Retrieved 2010-11-02

[9]. The World Bank;Poverty-Overview;Retrieved October 20,2009

[10]. Mehrdad.M.;Subjective well being,Depression,Anxiety and Stress Ratings;A comparison of Healthy and Unhealthy Students;WHOQOL-BREF and DASS-42

Appendix i-

\section{Appendices}

$$
\begin{aligned}
& \mathrm{X}^{2}=\sum_{\text {row total } x \text { column total }} \frac{(\mathrm{o}-\mathrm{E})^{2}}{E} \\
& \mathrm{E}=\frac{\text { Grad total }}{82}=23.049 \\
& \mathrm{E}_{1}=\frac{45 x 42}{82} \\
& \mathrm{E}_{2}=\frac{45 x 40}{82}=21.951
\end{aligned}
$$


$\mathrm{E}_{3}=\frac{37 x 42}{82}=18.95$

$\mathrm{E}_{4}=\frac{37 x 40}{82}=18.05$

$\mathrm{d}_{\mathrm{f}}=(\mathrm{r}-1)(\mathrm{c}-1)$

$=(2-1)(2-1)$

$=1$

Appendix ii-

$\mathrm{E}=$ row total $x$ column total

$\mathrm{E}_{1}=\frac{42 x 42}{82}=21.51$

$\mathrm{E}_{2}=\frac{42 x 40}{82}=20.49$

$\mathrm{E}_{3}=\frac{42 x 40}{82}=20.49$

$\mathrm{E}_{4}=\frac{40 x 40}{82}=19.51$

$\mathrm{d}_{\mathrm{f}}=(\mathrm{r}-1)(\mathrm{c}-1)$

$=(2-1)(2-1)$

$=1$

Appendix iii

Where a component

Where a component

$\mathrm{E}_{1}=\frac{42 x 22}{82}=11.27$

$\mathrm{E}_{2}=\frac{42 \times 8}{82}=4.097$

$\mathrm{E}_{3}=\frac{42 \approx 15}{82}=7.68$

$\mathrm{E}_{4}=\frac{42 x 8}{82}=4.097$

$\mathrm{E}_{5}=\frac{42 x 17}{82}=8.71$

$\mathrm{E}_{6}=\frac{42 \approx 12}{82}=6.15$

$\mathrm{E}_{7}=\frac{40 x 22}{82}=10.73$

$\mathrm{E}_{8}=\frac{40 x 8}{82}=3.90$

$\mathrm{E}_{9}=\frac{40 x 15}{82}=7.32$

$\mathrm{E}_{10}=\frac{40 x 8}{82}=3.902$

$\mathrm{E}_{11}=\frac{40 x 12}{82}=8.29$

$\mathrm{E}_{12}=\frac{40 x 12}{82}=5.85$

Appendix iv:

$\mathrm{E}_{1}=\frac{40 x 22}{82}=10.73$

$\mathrm{E}_{2}=\frac{40 x 8}{82}=3.9$

$\mathrm{E}_{3}=\frac{40 x 15}{82}=7.32$

$\mathrm{E}_{4}=\frac{40 x 8}{82}=3.90$ 
$\mathrm{E}_{5}=\frac{40 x 17}{82}=8.29$

$\mathrm{E}_{6}=\frac{40 x 12}{82}=5.85$

$\mathrm{E}_{7}=\frac{42 x 22}{82}=11.27$

$\mathrm{E}_{8}=\frac{42 x 8}{82}=4.097$

$\mathrm{E}_{9}=\frac{42 \approx 15}{82}=7.68$

$\mathrm{E}_{10}=\frac{42 x 8}{82}=4.907$

$\mathrm{E}_{11}=\frac{42 \times 17}{82}=8.71$

$\mathrm{E}_{12}=\frac{42 \approx 12}{82}=6.15$

Appendix v

$\mathrm{E}_{1}=\frac{41 x 12}{74}=6.6$

$\mathrm{E}_{2}=\frac{41 x 9}{74}=4.99$

$\mathrm{E}_{3}=\frac{41 x 8}{74}=4.4$

$\mathrm{E}_{4}=\frac{41 \times 6}{74}=3.3$

$\mathrm{E}_{5}=\frac{41 x 14}{74}=7.7$

$\mathrm{E}_{6}=\frac{41 x 12}{74}=6.65$

$\mathrm{E}_{7}=\frac{13 x 41}{74}=7.20$

$\mathrm{E}_{8}=\frac{12 x^{33}}{74}=5.35$

$\mathrm{E}_{9}=\frac{9 x 33}{74}=4.01$

$\mathrm{E}_{10}=\frac{8 x 33}{74}=3.57$

$\mathrm{E}_{11}=\frac{6 x 33}{74}=2.67$

$\mathrm{E}_{12}=\frac{14 x 33}{74}=6.24$

$\mathrm{E}_{13}=\frac{12 x^{33}}{74}=5.35$

$\mathrm{E}_{14}=\frac{13 x 33}{74}=5.8$

Appendix vi

$\mathrm{E}_{1}=\frac{12 x^{38}}{74}=6.2$

$\mathrm{E}_{2}=\frac{9 x 38}{74}=4.62$

$\mathrm{E}_{3}=\frac{8 x 38}{74}=4.1$

$\mathrm{E}_{4}=\frac{6 x 38}{74}=3.08$

$\mathrm{E}_{5}=\frac{14 x^{38}}{74}=7.19$

$\mathrm{E}_{6}=\frac{12 \times 38}{74}=6.16$ 
$\mathrm{E}_{7}=\frac{13 x^{38}}{74}=6.67$

$\mathrm{E}_{8}=\frac{12 x^{36}}{74}=5.8$

$\mathrm{E}_{9}=\frac{9 x 36}{74}=4.38$

$\mathrm{E}_{10}=\frac{8 x 36}{74}=3.89$

$\mathrm{E}_{11}=\frac{6 x 36}{74}=2.92$

$\mathrm{E}_{12}=\frac{14 x^{36}}{74}=6.81$

$\mathrm{E}_{13}=\frac{12 x 36}{74}=5.84$

$\mathrm{E}_{14}=\frac{13 x 36}{74}=6.32$

Appendix vii

$\mathrm{E}_{1}=2.35$

$\mathrm{E}_{2}=11.76$

$\mathrm{E}_{3}=14.12$

$\mathrm{E}_{4}=5.29$

$\mathrm{E}_{5}=6.47$

$\mathrm{E}_{6}=1.65$

$\mathrm{E}_{7}=8.23$

$\mathrm{E}_{8}=9.88$

$\mathrm{E}_{9}=3.70$

$\mathrm{E}_{10}=4.53$

Appendix viii

$\mathrm{E}_{1}=2.11$

$E_{2}=10.6$

$\mathrm{E}_{3}=12.7$

$\mathrm{E}_{4}=4.76$

$\mathrm{E}_{5}=5.82$

$\mathrm{E}_{6}=1.88$

$\mathrm{E}_{7}=9.41$

$\mathrm{E}_{8}=11.29$

$\mathrm{E}_{9}=4.23$

$\mathrm{E}_{10}=5.18$

Appendix ix

$\mathrm{E}_{1}=3.32$

$\mathrm{E}_{2}=24.9$

$\mathrm{E}_{3}=11.08$

$\mathrm{E}_{4}=1.66$

$\mathrm{E}_{5}=2.67$

$\mathrm{E}_{6}=20.07$

$\mathrm{E}_{7}=8.919$

$\mathrm{E}_{8}=1.34$

$$
\begin{aligned}
& \text { Appendix x } \\
& \mathrm{E}_{1}=3.16 \\
& \mathrm{E}_{2}=23.7 \\
& \mathrm{E}_{3}=10.5 \\
& \mathrm{E}_{4}=1.58 \\
& \mathrm{E}_{5}=2.84 \\
& \mathrm{E}_{6}=21.28 \\
& \mathrm{E}_{7}=9.46
\end{aligned}
$$


$\mathrm{E}_{8}=1.42$

\section{Abbreviations}

QOL Quality of life

MCS Mental component score

PCS Physical component score

HMO Health Management Organisation

$\mathbf{X}^{2} \quad$ Chi square

Df

Degree of freedom= (number of rows-1) $\mathrm{x}$ (number of column-1)

$\mathrm{ACE}$

$\mathbf{P}$ Angiotensin-converting enzyme

(O)- (E) Observed -Expected

QALY Quality-adjusted life years

a

MC

Tabulated X2 at a specific significance level determined by the df Mama Cass

Ca

T\&B

CH

Cafeteria (School cafeteria)

Sc

$\mathrm{T} \& \mathrm{~B}$ canteen

Chills

Self cooking

O

Others 